only exon 15 that encodes the O-linked sugar domain (3). Neonatal diagnosis of FH in four fetuses from the family was possible through analyses of their LDLR genes in cord blood samples at delivery.

Another new variant of LDLR gene (FHTonami-2) with $10 \mathrm{~kb}$ deletion eliminating exons 2 and 3 was ascertained in 9 families (4). This mutant gene deleted the first and the second repeats of ligand binding domain of LDLR, which was confirmed by the absence of cross reactive material of monoclonal anti-LDLR antibody, IgG-C7, in the cultured skin fibroblasts of FH-Tonami-2 homozygotes. The affinity of this mutant receptor for LDL particle was approximately $40 \%$ of normal receptor. Serum cholesterol levels in FH-Tonami-2 patients were lower, in both homozygotes and heterozygotes, and two heterozygotes were found to be normocholesterolemic. Development of coronary atherosclerosis of FH-Tonami-2 was slower than that of classical FH patients. All 4 true homozygotes with FH-Tonami-2 are alive to reach their middle ages (62, 51, 48 and 33 years), and the heterozygotes also lived longer than classical FH patients. From these results, we conclude that FH-Tonami-2, caused by the partially impaired LDLR with small deletion in its ligand binding domain, produces a mild type of $\mathrm{FH}$.

\section{REFERENCES}

1) Mabuchi $H$, Miyamoto $S$, Ueda $K$, et al: Causes of death in patients with familial hypercholesterolemia. Atherosclerosis 61: 1, 1986.

2) Mabuchi H, Koizumi J, Shimizu M, et al: Development of coronary heart disease in familial hypercholesterolemia. Circulation 79: 225, 1989.

3) Kajinami $\mathrm{K}$, Mabuchi $\mathrm{H}$, Itoh $\mathrm{H}$, et al: New variant of low density lipoprotein receptor gene. FH-Tonami. Arteriosclerosis 8: 187, 1988.

4) Kajinami K, Fujita $H$, Mabuchi $H$, et al: Familial hypercholesterolemia with partial deletion in the ligand binding domain of low density lipoprotein receptor associated with mild hypercholesterolemia and normocholesterolemia: FH-Tonami-2. (Submitted for publication).

\title{
Supplementary (1) Three Mutations Including Two Deletions of the Low Density Lipoprotein Receptor Gene in Japanese Patients with Homozygous Familial Hypercholesterolemia
}

\author{
Tohru FUNAHASHI, Yasuko MiYAKE and Akira YAMAMOTO
}

Department of Etiology and Pathophysiology, National Cardiovascular Center Research Institute, Suita

Mutation of the low density lipoprotein (LDL) receptor in familial hypercholesterolemia $(\mathrm{FH})$ is heterogeneous. Multiple mutations disrupt the function of LDL receptor. We here report the new mutations of the LDL receptor gene in 22 Japanese patients with homozygous FH.

Method: 1) LDL receptor activity was measured by the method of Brown and Goldstein. After the skin fibroblasts from the patients were cultured with lipoprotein deficient serum for $48 \mathrm{~h}$, the cells were incubated with ${ }^{125} \mathrm{I}$-LDL and the amount of bound ${ }^{125}$ I-LDL was counted. 2) Bio- synthesis of LDL receptor protein was observed by the method of Tolleshaug et al. After the cells were labeled with $\left[{ }^{35} \mathrm{~S}\right]$ methionine, LDL receptor protein was immunoprecipitated with anti-LDL receptor antibody (IgG C7). Immunoprecipitates were subjected to SDS polyacrylamide gel electrophoresis and detected by fluorography. 3) Rearrangement of LDL receptor gene was analyzed by Southern blotting. Genomic DNA was prepared from whole blood cells or fibroblasts from the patients. After digestion with endonuclease, DNA was transferred to nylon membrane filter and hybridized with LDL receptor 
cDNA probe (pLDL R3).

Results: 1) No immunodetectable receptors were detected in five patients $(23 \%)$. Southern blotting showed no apparent rearrangement in the LDL receptor gene and LDL receptor mRNA with normal size could be detected in all the patients. But the expression levels were extremely low. Three patients $(60 \%)$ in this group affected coronary artery disease (CAD). 2) Mutant LDL receptors with abnormal size were synthesized in five patients $(23 \%$, three kindreds). The first mutant synthesized $155 \mathrm{kD}$ receptor with rapid degradation. Southern blotting could not detect the abnormality of this mutant gene. The second mutant synthesized $150 \mathrm{kD}$ receptor, which was gradually secreted into the medium. Fibroblasts of the patients bound LDL but failed to internalize it. A $7.2 \mathrm{~kb}$ deletion was present in 3' half of the LDL receptor gene which en- codes membrane spanning and cytoplasmic domain of the receptor. The third mutant synthesized $120 \mathrm{kD}$ receptor which failed to bind LDL but could bind $\beta$ VLDL. A $12 \mathrm{~kb}$ deletion was detected in her LDL receptor gene. Three patients with the first mutation and a patients with the second mutation suffered from CAD. 3) Twelve patients (54\%) synthesized a significant amount of the LDL receptor with normal size. In 6 patients the LDL receptor activity was $20-30 \%$ of normal values and it was below $10 \%$ of normal in the other 6 patients. Nine patients $(75 \%)$ of this group could avoid CAD.

Conclusion: Mutations of the LDL receptor gene in Japanese FH was heterogeneous. Three types of peculiar mutant phenotypes including two deletions were found. About half of the patients synthesized normally appearing receptor and the incidence of CAD was low in this group patients.

\title{
Supplementary (2) Defective Low Density Lipoprotein Receptors and Atherosclerosis
}

\author{
Kenji ISHII and Toru KITA
}

Department of Geriatric Medicine, Faculty of Medicine, Kyoto University, Kyoto

Low density lipoprotein (LDL) plays important roles in both cholesterol transfer and atherosclerosis. The biochemistry and genetics of the LDL receptor are now well understood. However, much less is known about how the plasma level of LDL increases in familial hypercholesterolemia $(\mathrm{FH})$ patients, and how the elevated level promotes atherosclerosis. An animal model is necessary to answer these questions. The Watanabe heritable hyperlipidemic (WHHL) rabbit has a genetic defect that is identical to that of FH in humans. In the homozygous form, this defect leads to a nearly complete deficiency of LDL receptors. As a result, the plasma cholesterol level in these rabbits is elevated 10 - to 20 -fold above normal. LDL deposits in WHHL rabbit tissue produce xanthomas and atheromas.

Studies of WHHL rabbit have highlighted the important part that LDL receptors in the liver play in controlling both the production and the clearance of lipoproteins that carry endogenous cholesterol. Changes in the number of hepatic LDL receptors cause reciprocal changes in LDL synthesis and degradation, resulting in changes in plasma LDL levels. Deficient hepatic LDL receptors, as found in the WHHL rabbit, result in overproduction and undercatabolism of LDL. This knowledge leads to theoretical plan to treat hypercholesterolemia. The therapeutic implications of the LDL receptor studies center on strategies for increasing the production of LDL receptors in the liver, thereby lowering plasma LDL-cholesterol levels. In combination of inhibitor of 3-hydroxy-3-methylglutaryl coenzyme A reductase, the rate-limiting enzyme of de novo synthesis of cholesterol in the liver, and bile acid adsorbent resin, we successfully induced hepatic mRNA for $\mathrm{LDL}$ receptors and the receptor protein in 